\title{
TRIZ-Based Patent Investigation by Evaluating Inventiveness
}

\author{
Daniele Regazzoni ${ }^{1}$ and Roberto Nani $^{2}$ \\ ${ }^{1}$ University of Bergamo, viale Marconi, 24044 Dalmine (BG), Italy \\ ${ }^{2}$ Scinte Srl, Ranica (BG), Italy
}

\begin{abstract}
Among TRIZ users the importance given to patent resources is far behind the mere protection of $R \& D$ results. Patents represent a starting point for new inventions and a huge resource for collecting information on the way contradictions have been solved and in which different field such solutions may be adopted. Moreover the worldwide patent database contains information about the technology evolution that can be extracted so that the level of maturity of a product or process can be evaluated towards TRIZ laws of technical evolution. The contribution of this paper is to provide a step by step procedure, partially automatic, to perform TRIZ oriented patent search. The procedure, while determining the level of innovation of patents, allows defining a sharp set of patents responding to a structured query. The procedure has been applied to several case studies of different fields (e.g. mechanical, medical, and electronics) and a significant example referring to X-rays technology is shown in the paper.
\end{abstract}

Keywords: patent investigation, TRIZ, level of inventiveness, patent clustering

\section{Introduction}

This paper presents a systematic approach to search and classify world patents in groups according to their degree of novelty and inventiveness. TRIZ methodology has been used to define the criteria to discriminate patents describing a small inventive step or optimization from real breakthrough inventions and discoveries. TRIZ literature presents a large number of papers referring to the subject of level of innovation [1-3] as it is part of basic concepts of the methodology, and patent search methods according to TRIZ are widely known as well. By the way usually the focus is put on the way patents can be exploited to innovate more while the previous step, finding the right set of patents, is less considered. This may cause the whole work to fail because some information are missing, or not enough time is available to perform manual analyses.

Please use the following format when citing this chapter: 
The goal of the overall research is to provide the inventor with a procedure to perform better and quicker patent search. Then the results obtained could be used for several purposes, such as:

- Defining the state of the art of technologies from emerging to mature;

- Assessing the degree of inventiveness of a patent or concept;

- Building quantitative curves describing main drivers trends of a technology;

- Forecasting future evolution of technology and products.

Ikovenko et al. [4] define five steps to enhance Intellectual Property (IP) strategy taking advantage of TRIZ tools: evaluate goals and area to protect, evaluate company assets, develop a defensive patent strategy, implement it to build broad patents and, finally, manage your patent portfolio. TRIZ together with problem analysis and semantic tools are powerful instruments for patent strategies development. It is necessary to further sharpen algorithms and recommendations on using specific TRIZ tools for specific strategic IP objectives.

TRIZ practitioners are familiar with using patent resources to perform innovation in addition to protection and circumvention. Generally patents are required to be classified by the Contradiction they solved and Inventive Principles they used instead of the fields in which they are involved. Most of the currently available general purpose patent classification systems, such as International patent Classification or European Classification, are based on technology-dependent schemes and they hardly satisfy TRIZ users' requirements. TRIZ-oriented automatic systems to identify Contradictions and Principles have been developed and notable enhancements have been obtained in the last years $[5,6]$ but they are not yet mature for a wide diffusion.

\section{Methodology}

The methodology presented in this paragraph has not the goal to obtain better results in terms of patent found compared to other TRIZ based patent search method. What is claimed is a better usability and short timing in gathering the desired set of patents. This goes through a less iterative procedure in which there is almost no need to read the content of any considered patent. The methodology takes into consideration Souchkov's works [7] on the classification of five Levels of Invention and on the differentiations of solutions according to such classification. Souchkov distinguishes novelties of solutions on the base of three criteria:

1. Function: Any man-made system is designed to fulfil a certain purpose, which, in turn, is provided by a certain main function of the system;

2. Principle: This is a basic scientific effect, principle or phenomenon that enables function to be delivered. 
3. Market: Any man-made system delivers its function within a certain context, which meets a certain need of a specific market.

The methodology developed determines a model capable to (a) classify every man-made system on a function-principle combination and (b) individuate the systems and sub-systems that materialize such function-principle combination. For what concerns market no information are retrieved by patent database and market issues will not be taken into consideration.

The step by step procedure explained in the followings is based on an intensive use of Delphion [8] patent search engine capable of:

1. Browsing Codes according to International Patent Classifications, using keyword searching to find relevant IPC classes going down through the entire hierarchy. At each level of the hierarchy the number of child classes is identified, along with the number of patents associated with each class.

2. Clustering Texts to examine search results using linguistic technologies to explore relationships among patents creating a more targeted analysis of patent data and analyzing similarities to focus on what's most relevant.

\subsection{Patent Investigation and Analysis Procedure}

The procedure is composed of five steps as shown in the scheme of Figure 1. Starting from a research context or general interest in a technology the procedure gives back small and precise sets of patents depending on specific objective of the research project, such as cost reduction, elimination of a device or change in the product layout. In the following each step is explained in details while the next paragraph shows step by step the application to the X-ray technology and results are discussed.

\subsubsection{Step 1: Boolean search}

On the base of a given subject or field of research the first step consist in performing a Boolean search using few and generic keywords defining the technology, and no restriction on the IPC (International Patent Classification) classes are imposed. The search results consist in a wide set of patents describing several different aspects of the technology and commonly distribute over several different fields of application. After the search algorithm has been defined and results are given from the patent database some simple statistical elaborations are made. Starting from the date of the first patent of interest some trends are plotted over time to describe:

- The number of patents filed per year;

- The cumulative number of patents filed per year

- The number of IPC 4 digit classes in which patent have been classified per year 


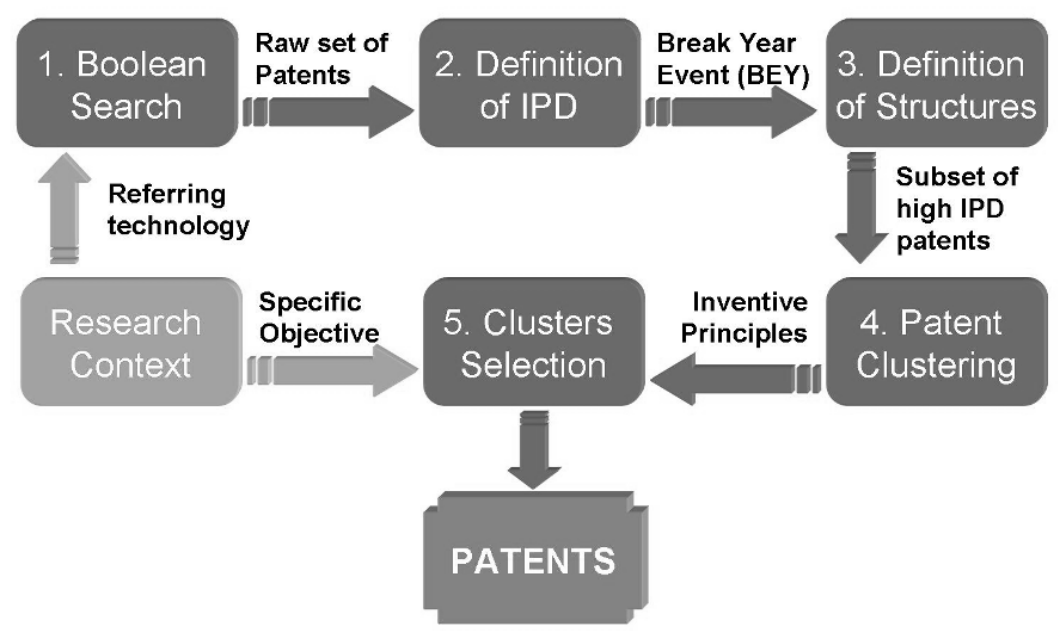

Fig. 15 steps scheme of the patent investigation and analysis procedure

Although data are still to be refined and analyzed according to TRIZ tools, the shape of the cited graphs is a preliminary raw indicator of the level maturity of the technology searched. In particular the cumulative number of patents may resemble to a complete or partial $\mathrm{S}$ curve and hypothesis on the stage of evolution can be done.

\subsubsection{Step 2: Definition of Intellectual Property Density (IPD)}

The second step consists in the definition of the Intellectual Property Density (IPD) given by the ration of number of patents over the number of IPC 4 digit classes per year. The IPD indicator shows the concentration of patents of the given technology according to the IPC classification. Plotting the cumulative graph of the Intellectual Property Density the Break Year Event (BYE) can be defined. The cumulate IPD is a simple but effective tool to discriminate low and high level innovation patents on the base of the stage of evolution. Actually, as it is commonly recognized that inventive level of patents applications decreases along the $\mathrm{S}$ curve, we can roughly divide patents before and after a specific year named BYE. Finding the BYE is necessary for the following steps but its determination cannot be performed universally by means of an automatic algorithm. A technician with some expertise in patent analysis may recognise the BYE by observing the graph cited before. It may correspond to the change of concavity in the cumulative density of IP, or to specific small signals. For emerging technology the last stages of evolution are not been reached and all patents may belong to the first group. Any- 
way, a mistake of a few years can be generally tolerated since it does not impact dramatically on the overall results.

\subsubsection{Step 3: Structures of Novelty}

A direct result of the definition of the BYE is the creation of two subset of patents that will be addressed as Structure A and B. Structure A is made by the patents filed before the BYE that are characterized by a low level of intellectual property density. Patents of Structure B on the contrary are subsequent to the BYE and belong to several different IPC classes. According to TRIZ classification of level of invention we can assume inventions of level 1-2 belongs to Structure B while inventions of level 3-4-5 belong to Structure A. The subset of patents of Structure A and B can be easily obtained by opportunely adding a time frame to the search algorithm of step 1 .

\subsubsection{Step 4: Clustering}

In step 4 a clustering algorithm is applied to patents of Structure A. Analyzing the text of patents terms are grouped in order to obtain the minimum number of cluster having almost no cross connections. The result can be automatically shown by a graph. Each cluster is composed by a number of terms, describing both devices and actions that for Structure A are quite homogeneous for meaning and field. A quick scan of the terms is enough to associate TRIZ Inventive Principles to each cluster. By the way there no need to go through all the clusters if in the working context a specific goal is defined. Actually, the goal can be directly associated to one or a few clusters and further analysis can be focused on those ones.

\subsubsection{Step 5: Functions and Systems}

The last step has the goal to identify a small set of highly interesting patents of the given technology responding to the specific goal of the research project. Once one or a few clusters of Structure A have been identified, Inventive Principles are used as keywords in a new search on Structure B. This allows highlighting critical components and interactions focused on the specific research goal. The overall output of the procedure consists in a list of patents strictly related on the research issues, together with a sharp indication on devices and functions involved.

\subsection{Application to X-rays technology}

X-rays technology has been chosen to demonstrate a real application of the procedure described so far. According to Step 1 the Boolean text search by words or 
phrases (alphabetical or numeric), using up to four designated patent fields, is performed. The search criterion (1) is:

$$
((\mathrm{x} \text {-rays })<\text { in }>\text { (TITLE,ABSTRACT,CLAIMS)) }
$$

Collections searched: European (Applications - Full text), European (Granted Full text), US (Granted - Full text), WIPO PCT Publications (Full text), US (Applications - Full text), 15,601 matches found of 11,254,955 patents searched

The patent data base gives the information (plotted in Figures 2-4), distributed on the Field Years, valid for European (Applications - Granted), US (Applications - Granted) and WIPO PCT Publications.

In the second step the distribution of patents in respect to the branches of technology is considered. In particular the Intellectual Patent Density is defined and plotted in Figure 5 and 6.

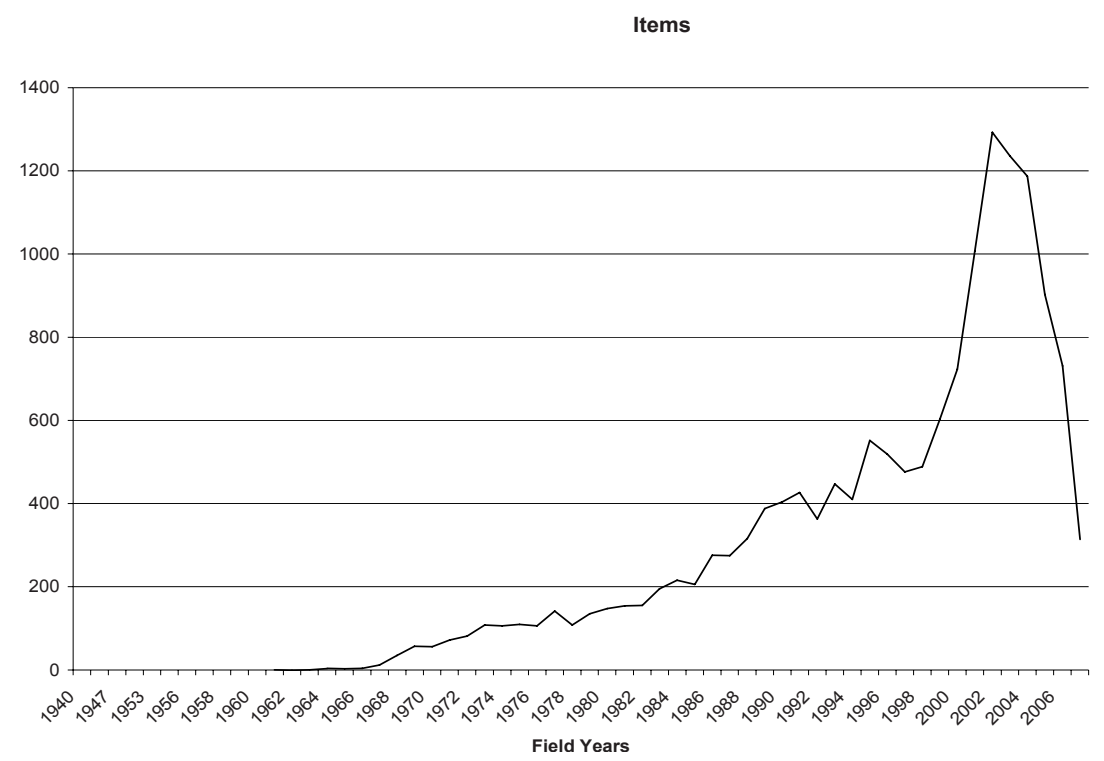

Fig. 2 Items: number of patent applications filed every year 


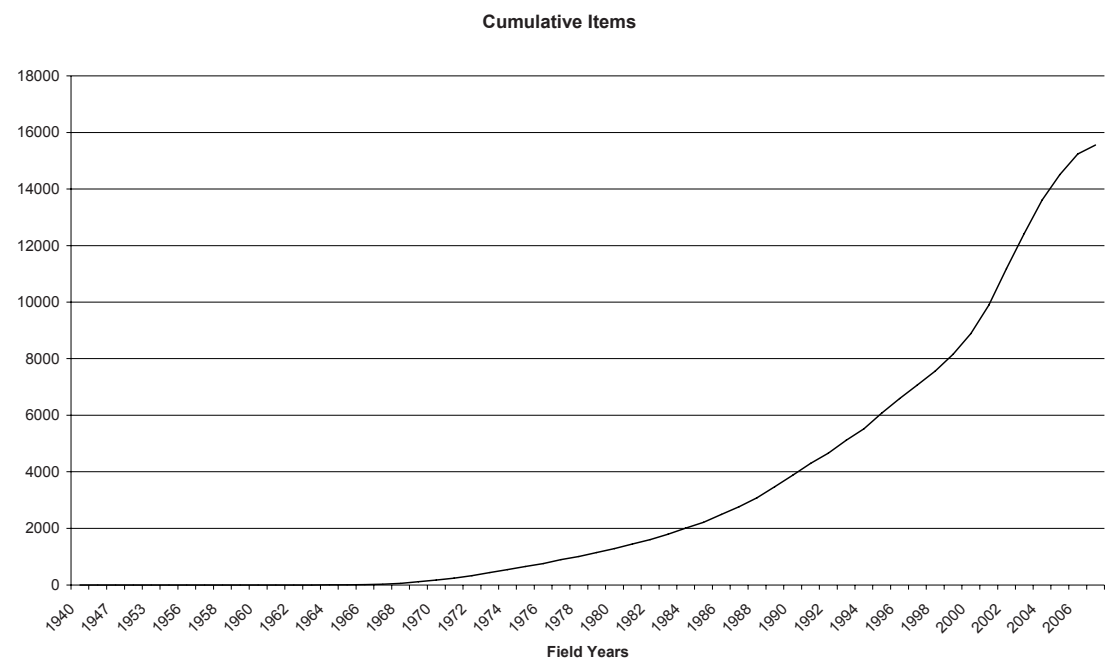

Fig. 3 Cumulative Items: number of the cumulated patents per year

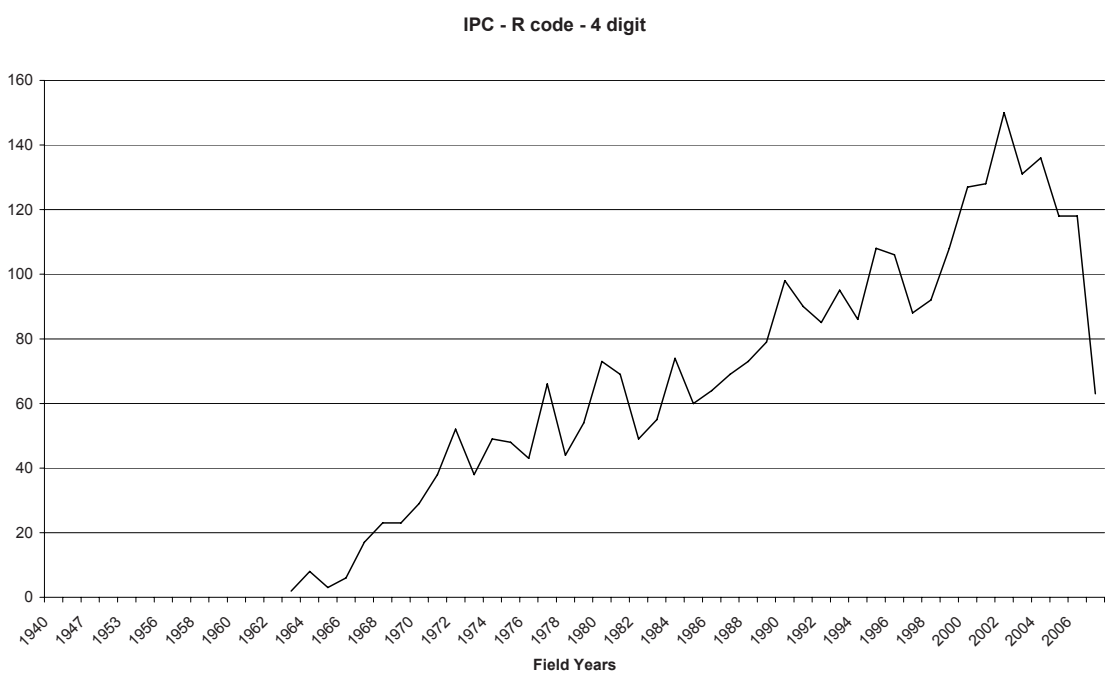

Fig. 4 IPC-R code: number of technological classes (expressed by 4 digit) engaged by the patents 
Items / IPC

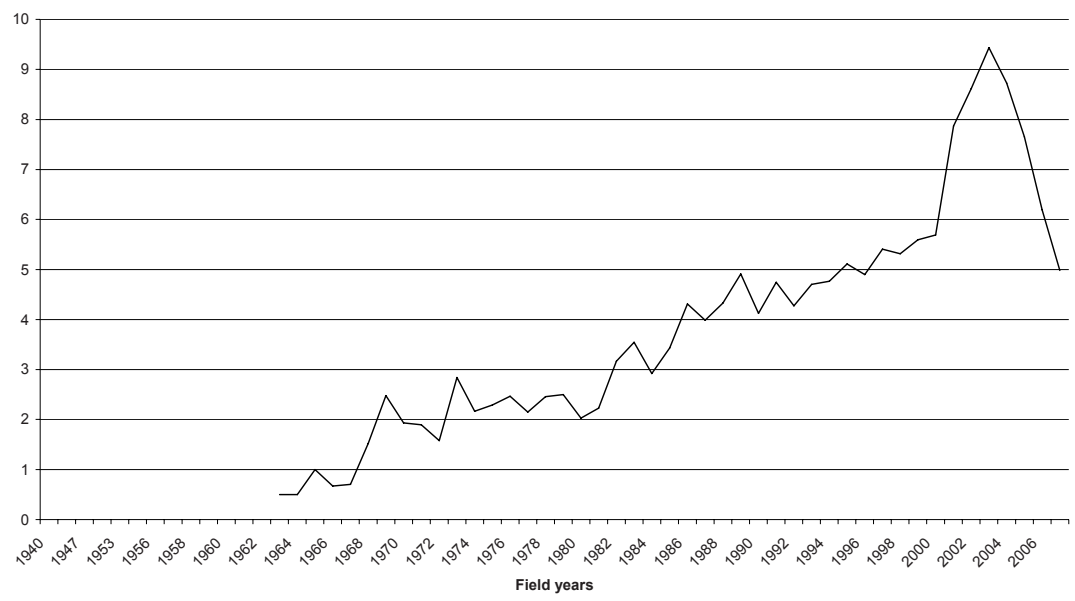

Fig. 5 IPD per year (Patents/IPC 4 digit per year)

Cumulative Items / Cumulative IPC

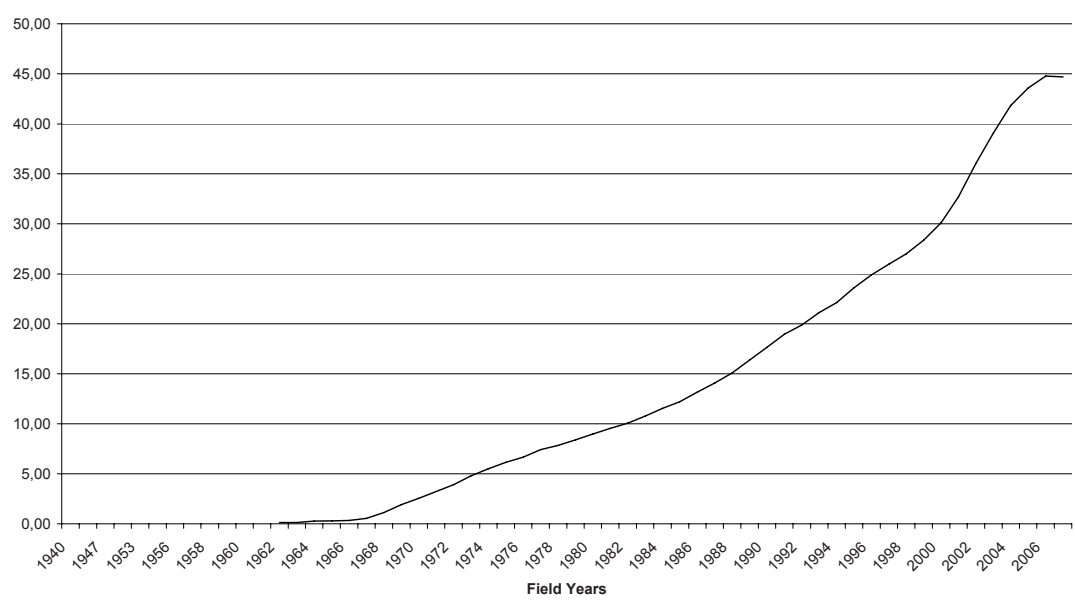

Fig. 6 Cumulative IPD (Cumulative Items/Cumulative IPC-R code per year)

In this case the Break Event Year (BYE) is simply determined as the year corresponding to the average value of the cumulative density of intellectual property. Thus, being 16 patents per IPC class the cumulative IPD average the BYE correspond to year 1989 .

Third step is aimed at classifying the whole set of patents into two Structures: 
- Structure A comprehend solutions of level 3,4 and 5: discovery, pioneering, radical creation of news functions and new systems;

- Structure B involves Level 1 and 2: relatively simple modification on the existing function/principle combination, relatively simple modifications of the existing subsystems, no new functionality, improving or merging existing solutions. Structure A is characterized by a low density of intellectual property.

In fact, every new patent application - regarding a discovery of a new principle (Level 5), or a creation of a radically new function/principle combination (Level 4), or the extending a function/principle combination (Level 3) - involves news IPC code, maintaining a low value of Density of Intellectual Property (IPD) and/or Cumulative Density of intellectual property.

Structure B is characterized by a high density of intellectual property. In fact, every new patent application - regarding a qualitative (Level 2) or quantitative (Level 1) improvement within existing function/principle/market - does not involve new IPC code. The two subsets of patents can be obtained by adding a time constraint to the Boolean algorithm used in step 1. Structure A is defined by: (X-rays) $<$ in $>$ (TITLE,ABSTRACT,CLAIMS) AND (DP $>=1960-01-01)$ AND $(\mathrm{DP}<=1989-12-31)$

Patents of Structure B are defined analogously.

Step 4 has to goal to create clusters and associate them with Inventive Principles. We take into consideration a number of clusters capable to involve only the principles avoiding or minimizing the number of connection of descriptive words between different clusters. A Text clustering of Structure A results to be optimized for 20 clusters for which there are only three combined clusters $(6 \leftarrow 10 \rightarrow 8)$ as shown in Figure 7.

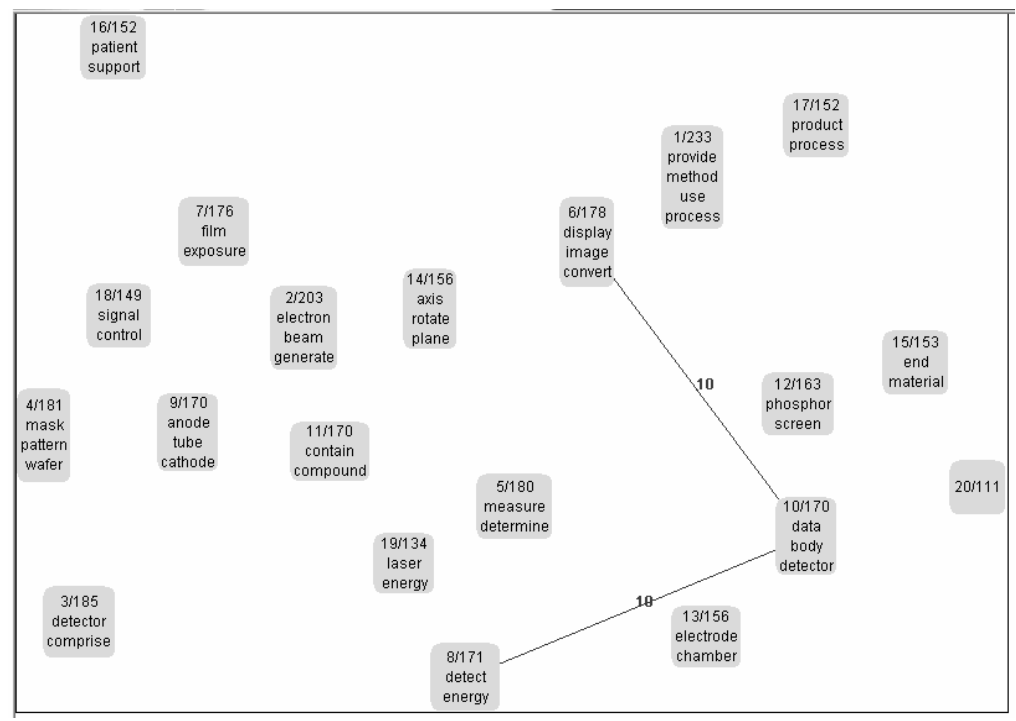

Fig. 7 Graphical representation of Clusters of Structure A and their limited interactions 
Table 1 Results of text clustering on Structure A

\begin{tabular}{|c|c|c|}
\hline$\underline{\text { Cluster }}$ & Items & Descriptive words \\
\hline 1 & 233 & $\begin{array}{l}\text { provide, method, use, process, disclose, comprise, form, } \\
\text { invention, radiation ( } 38 \text { ), material }\end{array}$ \\
\hline 2 & 203 & $\begin{array}{l}\text { electron, beam, generate ( } 25) \text {, target, electron beam, } \\
\text { apparatus, mean, focus, produce, source ( } 25)\end{array}$ \\
\hline 3 & 185 & $\begin{array}{l}\text { Detector (3), comprise, source ( } 25) \text {, detect, system, } \\
\text { device, plurality (1), apparatus, radiation (38), dispose }\end{array}$ \\
\hline$\cdots$ & $\ldots$ & $\ldots, \ldots$ \\
\hline 18 & 149 & $\begin{array}{l}\text { signal, control, (23) voltage, current, value, output, tube, } \\
\text { include, produce, predetermine }\end{array}$ \\
\hline 19 & 134 & $\begin{array}{l}\text { laser, energy, target, focus, use, produce, emit (38), beam, } \\
\text { obtain, high }\end{array}$ \\
\hline 20 & 111 & $\begin{array}{l}\text { Image (26), display, enable, use, provide, scan, film (30), } \\
\text { control (23), cause, mean }\end{array}$ \\
\hline
\end{tabular}

Step 5 prescribes the Boolean combination of items of structure B and principles extracted by the clustering texts of items of structure A. This allows to define the matching system and sub-system designed to fulfil a certain technical purpose. We consider for example the principles characterizing cluster 20 of structure A. We consider 10 clusters as optimized structure.

Table 2 Final set of clusters defining systems and subsystems of a technology

\section{Cluster Items Descriptive words}

120 comprise, carry, relate, form, process, move, invention, film, method, use

217 correspond, apparatus, first, light, method, receive, response, describe, capable, portion

912 patient, move, first, position, calculate, subject, detector, combine, tube, disclose

1011 utilize, object, display, radiation, source, digital, electronic radioscopic imaging system use, portable, pulse, self-contained 
We can observe that every cluster obtained is constituted by concatenated subclusters. Figure 8 shows cluster 1 that is formed by concatenated sub-clusters. The main sub-clusters contain the main function of the system.

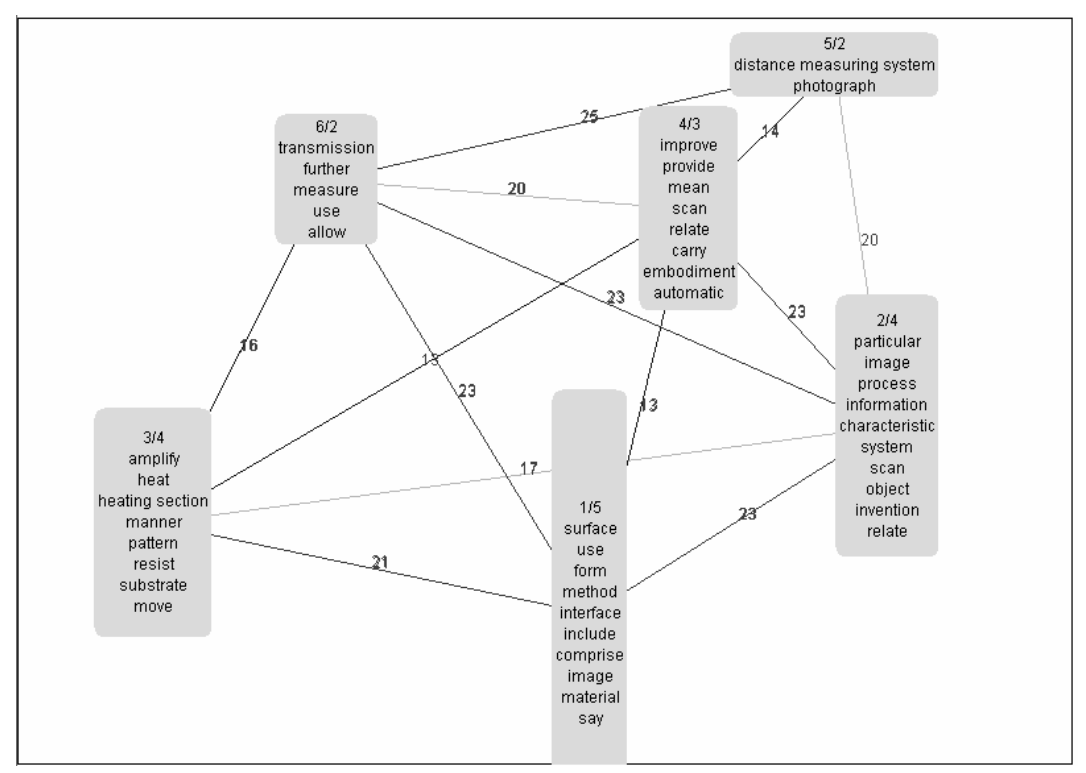

Fig. 8 Graphical representation of sub-Clusters of Structure Band their strong interactions

At last to each sub-cluster a small amount of highly focused patents are identified. For sub-cluster 1 five patents are found with a high level of similarity.

Table 3 Set of papers resulting from the patent search

\begin{tabular}{|c|c|}
\hline Publication & $\underline{\text { Similarity }}$ \\
\hline WO09930486A2 & $\begin{array}{l}\text { 59\% FORMING AN ASSEMBLED } \\
\text { SUCCESSIVE X-RAY IMAGES }\end{array}$ \\
\hline WO00243856A2 & $\begin{array}{l}\text { 55\% ORDERED TWO-AND THREE-DIMENSIONAL } \\
\text { STRUCTURES OF AMPHIPHILIC MOLECULES }\end{array}$ \\
\hline US26032433A1 & $\begin{array}{l}51 \% \text { Rapid X-ray diffraction method for structural analysis of a } \\
\text { nano material }[\ldots]\end{array}$ \\
\hline US26131669A1 & $49 \%$ Thin film transistor for imaging system \\
\hline WO09821625A2 & $49 \%$ WIDE LATITUDE FILM \\
\hline
\end{tabular}




\section{Conclusions}

The contribution of this paper to the state of the art of TRIZ oriented patent search is to provide a step by step procedure, almost automatic, based on level of invention. The described procedure allows to define a narrow set of patents responding to a structured query taking into account both general definition of a technology and a specific objective of research. The procedure is based on the advanced use of a patent search engine together with some TRIZ tools to gather results suitable for innovation means.

\section{References}

1. Gibson, N.: The Determination of the Technological Maturity of Ultrasonic Welding, The TRIZ Journal, http://www.triz-journal.com/archives/1999/07/a/index.htm (1999).

2. Leon, J., Martinez, C. Castillo: Methodology for the Evaluation of the Innovation Level of Products and Processes, proceedings of TRIZCON05, Brighton MI USA, April 2005 (2005).

3. Slocum, M. S., Lundberg, C. O.: Case Study: Using TRIZ to Forecast Technology, The Triz Journal, http://www.triz-journal.com/content/c070507a.asp (2007).

4. Ikovenko, S., Kogan, S.: TRIZ Application for Patent Strategies Development, Proceedings of TRIZ Future Conference p. 427-434, Graz, Austria, Nov. 16-18 (2005).

5. Han Tong Loh, Cong He and Lixiang Shen: Automatic classification of patent documents for TRIZ users World Patent Information, vol. 28, pp. 6-13, Issue 1, March 2006 (2006).

6. Han Tong Loh, Cong He: Grouping of TRIZ Inventive Principles to facilitate automatic patent classification Source. Expert Systems with Applications: An International Journal, vol. 34, Issue 1 (January 2008), ISSN:0957-4174 (2008).

7. Souchkov, V.: Differentiating Among the Five Levels of Solutions. The Triz Journal, http://www.triz-journal.com/archives/2007/07/02/ (2007).

8. www.delphion.com 